\title{
Declined Maternal GDCA is Correlated With Insulin Resistance and $\beta$-Cell Compensation in GDM: A Cross-Sectional Study in Chinese Pregnant Women
}

Bo Zhu

Zhejiang University

Zhixin Ma

Zhejiang University

Yuning Zhu

Zhejiang University

Lei Fang

Zhejiang University

Hong Zhang

Zhejiang University

Hongwei Kong

Dalian Institute of Chemical Physics

Dajing Xia ( $\nabla$ dxia@zju.edu.cn )

Zhejiang University

Research

Keywords: Gestational diabetes mellitus, Bile acids, Insulin resistance, $\beta$-cell compensation

Posted Date: June 15th, 2020

DOI: https://doi.org/10.21203/rs.3.rs-34077/v1

License: (c) (i) This work is licensed under a Creative Commons Attribution 4.0 International License.

Read Full License 


\section{Abstract}

\section{Background}

Gestational diabetes mellitus (GDM) is characterized by glycemia and insulin disorders. Bile acids (Bas) have emerged as vital signaling molecules in glucose metabolic regulation. Bas change in GDM are still unclear, it exerts great significance to illustrate the change of Bas in GDM.

\section{Methods}

We organized GDM patient $(n=67)$ and normal pregnant women $(n=48)$ around the oral glucose tolerance test (OGTT) screening period, fasting serums were collected for the measurement of Bas. Clinical data were collected and Bas metabolism profiles were analyzed in the GDM and normal glucose tolerance (NGT). Delivery characteristics, delivery gestational age and infant birthweight were abstracted from medical record to illustrate the differences among the groups.

\section{Results}

GDM patients exert the distinctive features compared with NGT, including higher BMI, glucose, insulin (both fasting and OGTT) and HbA1c levels. GDM also gained higher insulin resistance index (HOMA-IR) and decreased $\beta$-cell compensation (Dlo). Total bile acids (TBA) remained stable, but glycodeoxycholic acid (GDCA) and taurodeoxycholic acid (TDCA) declined significantly in GDM. GDCA was inversely correlated with HOMA-IR and positively correlated with Dlo. There exists no obvious difference in clinical outcome between GDM and NGT. However, GDM patients with high HOMA-IR and low Dlo are inclined to suffering higher cesarean delivery rate and earlier delivery gestational age.

\section{Conclusions}

GDM with significantly increased insulin resistance and declined Dlo elevated the worse clinical outcome, GDCA could be a valuable biomarker to evaluate insulin resistance and Dlo in GDM.

\section{Background}

Gestational diabetes mellitus (GDM) is a common medical complication of pregnancy with short- and long-term health risks for the mother, developing fetus and offspring [1]. This includes the high likelihood of subsequent maternal type 2 diabetes (T2DM), and possible adverse cardiometabolic phenotypes in the offspring. Women with GDM have an increased incidence of hypertensive disorders during pregnancy, including gestational hypertension, preeclampsia, eclampsia and thus the need for preterm caesarean section [2-4]. Offspring born to mothers with GDM are at increased risk of multiple immediate complications, including macrosomia, preterm birth, injury, shoulder dystocia, and respiratory distress, etc. [5-7]. 
Insulin resistance (IR) progressed with the gestational advance in order to supply adequate energy for the growing fetus. The increased IR promotes endogenous glucose production and the breakdown of fat stores, resulting in an elevation in glycemia and free fatty acid concentrations[8]. The abnormal metabolic adaptations of IR in pregnancy always consequently lead to GDM. Bile acids (Bas) have also emerged as crucial signaling molecules in glucose metabolism via nuclear hormone farnesoid $\mathrm{X}$ receptor (FXR) and Takeda G protein receptor 5 (TGR5) signaling pathways [9]. Studies in rodents have shown that induction of Bas secretion postprandial, leads to stimulation of glycogen storage and inhibition of hepatic glycolytic and lipogenic gene expression in a FXR dependent manner [10,11]. Bas activation of FXR causes repression of enzymes involved in hepatic gluconeogenesis such as phosphoenolpyruvate carboxy kinase (PEP-CK) and glucose 6-phosphatase [12,13]. In addition to the direct regulation, Bas are also involved in glucose metabolism through insulin secretion.

TGR5 is differentially activated by (lithocholic acid) LCA > (deoxycholic acid) DCA > chenodeoxycholic acid (CDCA) > cholic acid (CA), activation of TGR5 promotes intestinal $L$ cells to secrete glucagon like peptide-1 (GLP-1) which acts on the pancreatic $\beta$-cells to stimulate insulin secretion [14]. FXR is differentially activated by CDCA > DCA > LCA > CA[15], although GLP-1 secretion is negatively regulated by FXR, TGR5 activation in $L$ cells occurs rapidly postprandial, whereas activation of FXR induces a more delayed response that requires transcriptional activation $[16,17]$. The research of interaction between Bas and insulin illustrated that insulin regulates Bas composition by regulating Bas 12 a-hydroxylase cyp8b1 through Fox01, serum insulin level is positive correlated with 12 a-hydroxylated Bas (CA, DCA, and their conjugated forms) concentration[18]. In healthy subjects, IR was associated with increased 12 ahydroxylated Bas, ratios of 12 a-hydroxylated/non-12 a-hydroxylated Bas were associated with key features of IR. In type 2 diabetes mellitus (T2DM) patients, TBA were nearly twofold increased compared with healthy subjects, although no disproportionate increases in 12 a-hydroxylated Bas was spotted[19].

We have recently show that Bas metabolism profile changed with gestation advance, specifically, unconjugated bile acids dominate during the second trimester, conjugated bile acids overwhelmed in the third trimester[20]. As Bas individuals exerts distinctive affinity with FXR and TGR5 and they play the different role in glucose metabolism, it is necessary to clarify whether the Bas profile changed in GDM and the clinical significance of such change.

The main purpose of this work was to verify the hypothesis that Bas metabolism profile changed in GDM and there exist a correlation between insulin metabolism and specific Bas components. To address this issue, we analyzed the fasting Bas metabolism profile in GDM and normal pregnant women during OGTT screening, and identified key Bas individuals changed in GDM, which laid the foundation for the assessment of insulin resistance and $\beta$-cell compensation using Bas.

\section{Methods}

\section{Study design and population}


A total of 67 GDM patients and 48 normal pregnant women with a maternal age of 23-44 and a gestational age 21-29 weeks were enrolled between May 2019 to October 2019 in Women's Hospital of Zhejiang University School of Medicine, China. Pregnant Women with preconceptional diabetes, infections, abnormal liver or kidney function and positive for HIV and hepatitis $\mathrm{C}$ antibodies were excluded from participation. Blood samples were obtained from all the participants after 8-14 hours of fasting. All the women underwent a fasting 75-g oral glucose tolerance test (OGTT). Based on OGTT results, we defined GDM according to International Association of the Diabetes and Pregnancy Study Groups (IADPSG) criteria [21]. Insulin and glucose levels during the OGTT were used to estimate HOMAIR, HOMA- $\beta$, insulin secretion (using the Stumvoll first-phase estimate) and insulin sensitivity (using the Matsuda index) [22-24]. Insulin secretion index and Matsuda index were multiplied to calculate the oral disposition index (Dlo), which assesses $\beta$-cell compensation for insulin resistance [25]. This study was carried out according to the declaration of Helsinki guidelines, all the participants provided informed consent to participate in the follow-up study, the research protocol was approved by the ethics committee of the Women's Hospital of Zhejiang University School of Medicine (grant number: IRB-20200015-R).

\section{Bile acids measurement}

The bile acids measurement procedure was referred in previously published methods with minor modifications[20]. Briefly, reference standards were commercially available from TRC Inc. (Toronto, Canada) and Sigma-Aldrich (St. Louis, USA). $100 \mu$ of the serum specimen, standard solutions and quality control was vortex mixed with $300 \mu$ of the stable isotope-labeled internal standard (IS) stock solutions. A total of $150 \mu \mathrm{l}$ of the supernatant was aspirated by the autosampler for LC-MS/MS analysis after centrifuging $10 \mathrm{~min}$ at $13,000 \mathrm{~g}$. Bas were separated using a UPLC BEH C18 column $(2.1 \mathrm{~mm} \times 100$ $\mathrm{mm}, 1.7 \mu \mathrm{m}$; Waters) with the $40^{\circ} \mathrm{C}$ column temperature. Multiple reaction monitoring (MRM) measurements of the bile acids were performed using individually optimized cone voltage and collision energy (Table S1)

\section{Statistical methods}

Mass spectrometric data was analyzed using Analyst software v1.6.0 (SCIEX, Framingham, USA). Student's $t$ test or Mann-Whitney $U$ test were used to evaluated the difference among groups for continuous variables. One-way analysis of variance (ANOVA) or Kruskal-Wallis test was conducted to compare the different for categorical variables. Bas data were log transformed for orthogonal partial least square discriminant analysis (OPLS-DA) using SIMCA-P (version 13.0, Umetrics, Sweden). All the statistical analyses were performed using GraphPad Prism 7.0. $P<0.05$ was considered to be statistically significant.

\section{Results}

\section{Baseline characteristics of the study population}


Compared with NGT, GDM patients had higher BMI $(P<0.0001)$. In biochemical indicators including triglyceride (TG), cholesterol (TCH), High-density lipoprotein (HDL), low-density lipoprotein (LDL), Glycated albumin (GAL), HemoglobinA1c (HbA1c), HbA1c and TG elevated significantly $(P<0.0001)$ in GDM. Although there was no statistical significance in insulin secretion within the NGT group, fasting insulin and insulin in OGTT over 1 hour and 2 hours in GDM patients was significantly increased $(P=0.0003, P=$ $0.0048, P<0.0001)$. In addition, GDM patients had a deficiency in insulin sensitivity $(P<0.0001)$ and worse $\beta$-cell compensation $(P<0.0001)$ than that of NGT (Table 1$)$. We used all the significantly changed parameters in table 1 for OPLS-DA calculations to evaluate differences in GDM and NGT groups. There was a distinct clustering pattern between samples from GDM and NGT individuals (Figure 1A). The variable importance in projection (VIP) score for the mentioned indicator showed that DloखMatsuda index, HOMA-IR, Stumvoll Phase I and insulin levels in OGTT contributed significantly as a principal component to separate the two groups (Figure 1B).

\begin{tabular}{|c|c|c|c|}
\hline & NGT & GDM & \\
\hline & Median (IQR)/n (\%) & Median (IQR)/n (\%) & $P$ \\
\hline $\mathrm{N}$ & 48 & 67 & \\
\hline Age & $30(28-32.8)$ & $33(29-36)$ & 0.0029 \\
\hline Gestational age & $24(24-25)$ & $25(24-26)$ & 0.42 \\
\hline BMI $\left(\mathrm{kg} / \mathrm{m}^{2}\right)$ & $22.26(21.17-23.07)$ & $24.44(22.19-26.58)$ & $<0.0001$ \\
\hline Fasting glucose (mg/dl) & $78.0(74.9-82.1)$ & $85.0(78.1-94.0)$ & $<0.0001$ \\
\hline 1-h glu cose OGTT (mg/dl) & $137.5(110.5-150.9)$ & 185.2(165.1-197.5) & $<0.0001$ \\
\hline 2-h glu cose OGTT (mg/dl) & $116.2(104.3-130.5)$ & $159.9(142-172.3)$ & $<0.0001$ \\
\hline Fasting insulin $(\mu \mathrm{U} / \mathrm{ml})$ & $6.2(4.9-8.5)$ & $8.9(6.5-11.9)$ & 0.0003 \\
\hline 1-h insulin OGTT $(\mu \mathrm{U} / \mathrm{ml})$ & $40.5(30.3-63.3)$ & $58.6(39.7-91.5)$ & 0.0048 \\
\hline 2-h insulin OGTT $(\mu \mathrm{U} / \mathrm{ml})$ & $45.7(31.1-57.7)$ & $67.9(46.5-109.0)$ & $<0.0001$ \\
\hline Insulin sensitivity (Matsuda) & 7.78 (5.84-10.33) & $4.58(3.33-6.30)$ & $<0.0001$ \\
\hline Insulin secretion (Stumvoll) & $981.5(865.1-1264.0)$ & $1054.0(636.4-1321.0)$ & 0.68 \\
\hline DIo & $7572(6584-9121)$ & 4409 (3628-5186) & $<0.0001$ \\
\hline HOMA-IR & $1.20(0.92-1.71)$ & $1.87(1.28-2.64)$ & $<0.0001$ \\
\hline НОМА- $\beta$ & 157.7 (130.6-234.5) & 150.3(111.8-194.0) & 0.69 \\
\hline AUC (insulin/glucose) & $5.15(4.31-7.22)$ & $5.83(4.11-9.31)$ & 0.13 \\
\hline $\mathrm{TG}(\mathrm{mmol} / \mathrm{L})$ & $2.00(1.76-2.52)$ & $2.32(1.89-2.88)$ & 0.0469 \\
\hline $\mathrm{TCH}(\mathrm{mmol} / \mathrm{L})$ & $5.96(5.25-6.63)$ & $5.68(5.06-6.55)$ & 0.21 \\
\hline $\mathrm{HDL}(\mathrm{mmol} / \mathrm{L})$ & $1.96(1.63-2.24)$ & $1.79(1.56-2.00)$ & 0.09 \\
\hline $\mathrm{LDL}(\mathrm{mmol} / \mathrm{L})$ & $2.97(2.54-3.61)$ & $2.74(2.34-3.13)$ & 0.07 \\
\hline GAL (\%) & $12.2(11.6-13.0)$ & $12.3(11.7-12.9)$ & 0.31 \\
\hline HbA1c (\%) & $4.9(4.8-5.1)$ & $5.1(4.9-5.3)$ & 0.0127 \\
\hline
\end{tabular}




\section{Table 1. Characteristics of women with NGT and GDM}

IQR, inter quartile range. Differences across the NGT and GDM group were compared using the Kruskal-Wallis test for continuous variables and Fisher exact test for categorical variables. Biochemical indicators including fasting glucose and insulin,1/2-h-glucose and insulin, triglyceride (TG), cholesterol (TCH), High-density lipoprotein (HDL), low-density lipoprotein (LDL), Glycated albumin (GAL), HemoglobinA1c (HbA1c) were also compared between GDM and NGT. Insulin and glucose levels during the OGTT were used to estimate insulin secretion (using the Stumvoll first-phase estimate) and insulin sensitivity (using the Matsuda index). These indices were multiplied to calculate the oral disposition index (DIo), which assesses $\beta$ cell compensation for insulin resistance. Insulin resistance index and $\beta$-cell function were calculated using fasting glucose $(\mathrm{mM}) \times$ fasting insulin $(\mu \mathrm{IU} / \mathrm{ml}) / 22.5$ and $20 \times$ fasting insulin $(\mu \mathrm{IU} / \mathrm{ml}) /[$ fasting glucose $(\mathrm{mM})-3.5$ ] formulation respectively. $P<0.05$ was considered to be statistically significant.

\section{Bile acids metabolism profiles in GDM and NGT}

As glucose and insulin metabolism discriminations exist between GDM and NGT, and Bas are known to participate in the glycemia homeostasis via direct and indirect pathways, we postulated that GDM and NGT could be distinguished by Bas profiles. However, from the OPLS-DA results, the two cohorts could not be separated (Data not show), even though total bile acid (TBA) decreased slightly in the GDM group (Figure 2A). This finding suggested that specific types of Bas may be more intimately involved in glycemia regulation. Indeed, 12a-hydroxylated Bas decreased obviously $(P=0.04)$, Non-12a-hydroxylated Bas remained stable in GDM (Figure 2B). Furtherly, in 12a-hydroxylated Bas individuals, we verified that the concentrations of the TDCA and GDCA in fasting serum decreased significantly in the GDM cohort (TDCA, $P=0.03$; GDCA, $P=0.0261$ ), Non-12a-hydroxylated Bas individuals changed not significantly (Figure 2C). 
To further illustrate the relationship between Bas components and the obviously changed insulin related indicators, we analyzed the correlation of them. Fasting GDCA is positive correlated with insulin sensitivity in both NGT and GDM, the correlation coefficient decreased slightly in GDM. GDCA is also positively related to Dlo in GDM, but negatively with HOMA-IR and fasting insulin (FIN). TDCA has no correlation with insulin related indicators in NGT and GDM, although changed obviously in GDM. In non12a-hydroxylated Bas individuals, CDCA is positively related to HOMA-IR and FIN, TUDCA is negatively related Dlo (Figure 3).

\section{Clinical outcome of GDM and GDM with higher HOMA-IR and lower Dlo}

As GDCA declined in GDM and it is correlated with insulin sensitivity, IR, Dlo and FIN. In order to assess the relationship between GDCA and clinical outcome including delivery gestational age, fetus birthweight and cesarean delivery ratio, GDM patients were subdivided into four quartiles (Q1: $<25 \%, \mathrm{Q} 2: 25 \% \sim 50 \%$, Q3: $50 \% \sim 75 \%$ and Q4: $>75 \%$ ) according to the Matsuda index, HOMA-IR, Dlo and FIN respectively. GDCA changed not obviously among Q1 and Q4 groups divided by Matsuda index and FIN (Figure 4A, B), but decreased in HOMA-IR Q4 group and increased in Dlo Q4 groups (Figure 4C, D). In clinical outcome assess, GDM patients gained no variations compared with NGT, however, GDM with high HOMA-IR and decreased GDCA (HOMA-IR Q4) group exerts increased cesarean delivery ratio. GDM with low Dlo gained earlier delivery gestational age compared with NGT (Table2).

\begin{tabular}{|c|c|c|c|c|c|c|c|c|}
\hline & NGT & All-GDM & GDM Q1 (IR) & GDM Q4 (IR) & & GDM Q1 (DIo) & GDM Q4 (DIo) & \\
\hline & Median (IQR)/n (\%) & Median (IQR)/n (\%) & Median (IQR)/n (\%) & Median (IQR)/n (\%) & $P^{\#}$ & Median (IQR)/n (\%) & Median (IQR)/n (\%) & $P^{\#}$ \\
\hline $\mathrm{N}$ & $44 / 48$ & $59 / 67$ & $17 / 17$ & $12 / 17$ & & $15 / 17$ & $16 / 17$ & \\
\hline Missing data & 4 & 8 & 0 & 5 & & 2 & 1 & \\
\hline \multicolumn{9}{|l|}{ Delivery } \\
\hline Cesarean delivery & $36.4 \%(16 / 44)$ & $54.2 \%(32 / 59) \S$ & $41.2 \%(7 / 17) \S$ & $91.7 \%(11 / 12)^{* * *}$ & 0.008 & $66.7 \%(10 / 15) \S$ & $43.8 \%(7 / 16) \S$ & 0.2852 \\
\hline Gestational age & $39.3(39-41.1)$ & $39(38-39.6) \S$ & $39(38.4-39.3) \S$ & $38.6(37.4-39.4) \S$ & 0.718 & $38.4(37-39.3) *$ & $39(38.3-39.4) \S$ & 0.0733 \\
\hline Infant birth weight (g) & $3300(2970-3635)$ & $3400(3090-3670) \S$ & $3200(2950-3430) \S$ & $3430(3098-3980) \S$ & 0.1339 & $3400(2870-3810) \S$ & 3235 (3065-3428) § & 0.4453 \\
\hline
\end{tabular}

\section{Table 2. Delivery characteristics of women with NGT and GDM, GDM subgroups are divided by HOMA-IR and Dlo}

IQR, inter quartile range. Delivery characteristics including cesarean delivery rate, delivery gestational age and infant birth weight were compared in women with NGT, GDM and 
GDM subgroups. GDM patients were GDM patients were subdivided into four quartiles Q1, Q2, Q3, Q4) according to HOMA-IR and Dio, § represents no statistically significance with NGT group, $P^{\#}$ represents the difference across Q1 and Q4 groups. $P<0.05$ was considered to be statistically significant.

\section{Discussion}

In this study, we learned that the abnormalities of insulin-related indicators played the main role to distinguish GDM and NGT. Combined with the bile acids metabolism profile, we clarified the correlation between specific bile acids and insulin related indicators. Overall, GDM patients exert higher levels of BMI and $\mathrm{HbAc} 1$ during the OGTT screening period, in addition, fasting insulin and insulin at 1 and 2 hours post 75-g glucose intake increased significantly in GDM. In terms of bile acid metabolism profile, TDCA and GDCA were the most significantly changed individuals in GDM. Moreover, GDCA was negatively correlated with the insulin resistance index (HOMA-IR) and positively correlated with the $\beta$-cell compensation index (Dlo). In clinical outcomes assay, there was no significant difference between GDM and NGT, but GDM patients in HOMA-IR Q4 group possessed elevated ration of cesarean section delivery, and patients in Dlo Q1 group gained earlier delivery gestational ages. Therefore, our findings suggest that fasting GDCA levels during OGTT may be a useful indicator to evaluate insulin resistance and $\beta$-cell compensation, furtherly to predict adverse clinical outcomes of GDM.

GDM shares the similar pathogenesis with T2DM, $\beta$-cell function was insufficient to compensate for the need for glycemic homeostasis in pregnancy combined with reduced insulin sensitivity, finally result to increased serum glucose[1]. In addition to glucose and insulin metabolic disorders, lipids metabolism also changed in GDM, total triglycerides, total cholesterol, low-density lipoprotein cholesterol increase gradually throughout pregnancy in GDM[26]. Our results were consistent with previous report in clinical characteristic of GDM, including advanced age, higher BMI , TG and HbA1c.

Bile acids metabolism variation has been partially proved in T2DM, elevation of TBA increased the T2DM incidence, TBA almost doubled in T2DM patients compared with healthy subject[19,27]. Intrahepatic cholestasis of pregnancy (ICP) was characterized by an increased TBA, ICP women with higher TBA concentration are much more vulnerable to suffering GDM[28]. These studies suggest that TBA probably related to the occurrence of T2DM and GDM. Indeed, in Chinese prospective cohort studies, the incidence of GDM in the group with highest TBA $(\geq 4.0 \mu \mathrm{M})$ in early pregnancy had a 6.72 -fold increased risk of GDM compared with the lowest level. Even after adjusting potential confounders, TBA $\geq 2.0 \mu \mathrm{M}$ still presented an increased risk for developing GDM[29,30]. In our research, TBA changed slightly in GDM compared with normal glucose tolerance, this may be partially caused by the difference between the TBA detected by enzymatic cycling assay and the TBA consist of 15 bile acids individuals detected mass spectrometry. This provokes us that more attentiveness should payed to the bile acids components related to glucose metabolism. 
Research conducted on a large cohort of Chinese pregnant women in early pregnancy found that fasting serum levels of GUDCA $\leq 0.07 \mathrm{nmol} / \mathrm{mL}$ and DCA $\leq 0.28 \mathrm{nmol} / \mathrm{mL}$ were independently associated with an increased risk for development of GDM [31]. Untargeted metabolomics administrated in pregnant women's fasting serum during OGTT screening period spot that CA, ios-DCA and dehydio-LCA decreased in GDM[32]. In this research, GDCA and TDCA declined obviously in GDM. The difference between our research and the previous studies may be caused by the difference between targeted and non-targeted detection platforms and the samples collected in different trimesters, as our previous research has illustrated that bile acids metabolism profiles changed periodically with gestational age[20].

GDCA has no correlation with HOMA-IR and fasting insulin in normal glucose tolerance. In GDM, declined GDCA combined with the increased fasting insulin and fasting glucose result in the significant negative correlation between GDCA and HOMA-IR. Studies in vivo and in vitro demonstrated that GDCA is indeed related to insulin secretion and insulin resistance, more importantly, insulin resistance can be alleviated by GDCA administration. For example, fasting for 40 hours induce insulin resistance, while this insulin resistance model has no affect in bile acids metabolism profile. However, postprandial GDCA and insulin concentration changed in a significant positive correlation pattern. Increased GDCA triggered the secretion of insulin in a GLP-1 dependent manner[33]. To some extent, this is helpful to explain why even if GDCA elevated the post glucose intake in GMD patients, but declined baseline of GDCA still make it insufficient to promote insulin secretion via GLP-1, finally failed in the glycemia regulation. Polycystic ovary syndrome (PCOS) is usually accompanied by features of insulin resistance, HOMA-IR of PCOS patients is significantly negatively correlated with GDCA. Mechanistically, the significant increased Bacteroides vulgatus in the gut microbiota of PCOS individuals reduced the GDCA. GDCA combined with GATA-3 in the intestinal group 3 innate lymphoid cell to facilitate the interleukin-22 secretion, which in turn improves the PCOS phenotype including insulin resistance and ovarian function recovery[34]. However, whether variations occurred in GDM gut microbiome declined the GDCA and result in insulin resistance, the confirmation need further research.

Hyperglycemia burdened severely clinical outcome to both maternal and fetus outcome with GDM, but the clinical outcome of the whole GDM was not differed significantly from that of NGT in this study, which may be due to the early lifestyle intervention and even pharmacological therapy. Of note, GDM patients with serious deficiency of insulin compensation and high HOMA-IR subgroups gained worse clinical outcome.

Our study design, however, has several limitations. Firstly, the GLP-1 baseline was not measured in the fasting serum, and any variation in GLP-1 baseline in GDM and NGT may have influenced fasting insulin levels. Secondly, GLP-1 and Ba changes in 1-h and 2-h period after the glucose loading were also not detected, which limited the elucidation of the dynamic mechanism of Bas metabolism regulating insulin secretion through GLP-1, moreover, the fewer participants failed us to establish the cut-off point of GDCA, under which may increase the worse clinical outcome risk.

\section{Conclusions}


GDCA and TDCA declined significantly in GDM. GDCA was inversely correlated with insulin sensitivity and positively related with insulin compensation. which make it a valuable candidate biomarker for the assessment of insulin sensitivity and $\beta$-cell compensation. Declined GDCA is correlated to adverse pregnancy outcomes of GDM.

\section{Declarations}

\section{Ethics approval and consent to participate}

This study was carried out according to the declaration of Helsinki guidelines, the research protocol was approved by the ethics committee of the Women's Hospital of Zhejiang University School of Medicine (grant number: IRB-20200015-R). All the participants provided informed consent to participate in this study and the future follow-up study.

\section{Consent for publication}

Not applicable.

\section{Availability of data and materials}

All the data are available to interested researchers upon reasonable request. Requests for access to data should be made to the first author with e-mail:5202054@zju.edu.cn.

\section{Competing interests}

"\#" these authors have the same contribution to this article, none of the authors has any conflict in relation to the study

\section{Funding}

This work was supported by the National Key R\&D Program of China (Grant number: 2018YFC1002700, 2018YFC1002702).

\section{Author Contribution}

Bo Zhu and Zhixin Ma designed the study. Lei Fang and Hong Zhang collected the data. Zhixin Ma performed statistical data analyses. Bile acids measurement are conducted by Hongwei Kong. Yuning Zhu and Dajing Xia contributed to drafting the manuscript. All authors reviewed and edited the manuscript to produce the final version.

\section{Acknowledgement}

We thank all the doctors, nurses, technicians, especially the patients involved in their dedication to the study. 


\section{References}

[1] Johns EC, Denison FC, Norman JE, Reynolds RM. Gestational Diabetes Mellitus: Mechanisms, Treatment, and Complications. Trends in endocrinology and metabolism: TEM 2018; 29(11): 743-54.

[2] Group HSCR. Hyperglycemia and Adverse Pregnancy Outcome (HAPO) Study: associations with neonatal anthropometrics. Diabetes 2009; 58(2): 453-9.

[3] Wei Y, Yang H, Zhu W, Hod M, Hadar E. Adverse pregnancy outcome among women with pregestational diabetes mellitus: a population-based multi-centric study in Beijing. The journal of maternalfetal \& neonatal medicine : the official journal of the European Association of Perinatal Medicine, the Federation of Asia and Oceania Perinatal Societies, the International Society of Perinatal Obstet 2017; 30(20): 2395-7.

[4] O'Sullivan EP, Avalos G, O'Reilly MW, et al. Erratum to: Atlantic Diabetes in Pregnancy (DIP): the prevalence and outcomes of gestational diabetes mellitus using new diagnostic criteria. Diabetologia 2016; 59(4): 873.

[5] Mortier I, Blanc J, Tosello B, Gire C, Bretelle F, Carcopino X. Is gestational diabetes an independent risk factor of neonatal severe respiratory distress syndrome after 34 weeks of gestation? A prospective study. Archives of gynecology and obstetrics 2017; 296(6): 1071-7.

[6] Moll U, Landin-Olsson M, Nilsson C, Ursing D, Strevens H. Pregnancy outcome in women with gestational diabetes - a longitudinal study of changes in demography and treatment modalities. Acta obstetricia et gynecologica Scandinavica 2019.

[7] Yogev, Chen, Hod, et al. Hyperglycemia and Adverse Pregnancy Outcome (HAPO) study: preeclampsia. American journal of obstetrics and gynecology 2010; 202(3): 255 e1-7.

[8] Di Cianni G, Miccoli R, Volpe L, Lencioni C, Del Prato S. Intermediate metabolism in normal pregnancy and in gestational diabetes. Diabetes/metabolism research and reviews 2003; 19(4): 259-70.

[9] Shapiro H, Kolodziejczyk AA, Halstuch D, Elinav E. Bile acids in glucose metabolism in health and disease. The Journal of experimental medicine 2018; 215(2): 383-96.

[10] Watanabe $M$, Houten $S M$, Wang $L$, et al. Bile acids lower triglyceride levels via a pathway involving FXR, SHP, and SREBP-1c. The Journal of clinical investigation 2004; 113(10): 1408-18.

[11] Duran-Sandoval D, Cariou B, Percevault F, et al. The farnesoid $X$ receptor modulates hepatic carbohydrate metabolism during the fasting-refeeding transition. The Journal of biological chemistry 2005; 280(33): 29971-9.

[12] Zhang Y, Ge X, Heemstra LA, et al. Loss of FXR protects against diet-induced obesity and accelerates liver carcinogenesis in ob/ob mice. Molecular endocrinology 2012; 26(2): 272-80. 
[13] Potthoff MJ, Boney-Montoya J, Choi M, et al. FGF15/19 regulates hepatic glucose metabolism by inhibiting the CREB-PGC-1 alpha pathway. Cell metabolism 2011; 13(6): 729-38.

[14] Katsuma S, Hirasawa A, Tsujimoto G. Bile acids promote glucagon-like peptide-1 secretion through TGR5 in a murine enteroendocrine cell line STC-1. Biochemical and biophysical research communications 2005; 329(1): 386-90.

[15] Cariou B, Van Harmelen K, Duran-Sandoval D, et al. The farnesoid X receptor modulates adiposity and peripheral insulin sensitivity in mice. Journal of Biological Chemistry 2006; 281(16): 11039-49.

[16] Kim H, Fang S. Crosstalk between FXR and TGR5 controls glucagon-like peptide 1 secretion to maintain glycemic homeostasis. Laboratory animal research 2018; 34(4): 140-6.

[17] Trabelsi MS, Daoudi M, Prawitt J, et al. Farnesoid X receptor inhibits glucagon-like peptide-1 production by enteroendocrine L cells. Nature communications 2015; 6: 7629.

[18] Haeusler RA, Pratt-Hyatt M, Welch CL, Klaassen CD, Accili D. Impaired generation of 12-hydroxylated bile acids links hepatic insulin signaling with dyslipidemia. Cell metabolism 2012; 15(1): 65-74.

[19] Haeusler RA, Astiarraga B, Camastra S, Accili D, Ferrannini E. Human insulin resistance is associated with increased plasma levels of 12alpha-hydroxylated bile acids. Diabetes 2013; 62(12): 4184-91.

[20] Zhu B, Yin P, Ma Z, et al. Characteristics of bile acids metabolism profile in the second and third trimesters of normal pregnancy. Metabolism: clinical and experimental 2019; 95: 77-83.

[21] Weinert LS. International Association of Diabetes and Pregnancy Study Groups recommendations on the diagnosis and classification of hyperglycemia in pregnancy: comment to the International Association of Diabetes and Pregnancy Study Groups Consensus Panel. Diabetes care 2010; 33(7): e97; author reply e8.

[22] Stumvoll M, Van Haeften T, Fritsche A, Gerich J. Oral glucose tolerance test indexes for insulin sensitivity and secretion based on various availabilities of sampling times. Diabetes care $2001 ; 24(4)$ : 796-7.

[23] Stumvoll M, Mitrakou A, Pimenta W, et al. Use of the oral glucose tolerance test to assess insulin release and insulin sensitivity. Diabetes care 2000; 23(3): 295-301.

[24] Matsuda M, DeFronzo RA. Insulin sensitivity indices obtained from oral glucose tolerance testing: comparison with the euglycemic insulin clamp. Diabetes care 1999; 22(9): 1462-70.

[25] Elbein SC, Wegner K, Kahn SE. Reduced beta-cell compensation to the insulin resistance associated with obesity in members of caucasian familial type 2 diabetic kindreds. Diabetes care 2000; 23(2): 221-7. 
[26] Wang J, Li Z, Lin L. Maternal lipid profiles in women with and without gestational diabetes mellitus. Medicine 2019; 98(16): e15320.

[27] Zhu W, Wang S, Dai H, et al. Serum total bile acids associate with risk of incident type 2 diabetes and longitudinal changes in glucose-related metabolic traits. Journal of diabetes 2020.

[28] Martineau MG, Raker C, Dixon PH, et al. The metabolic profile of intrahepatic cholestasis of pregnancy is associated with impaired glucose tolerance, dyslipidemia, and increased fetal growth. Diabetes care $2015 ; 38(2)$ : 243-8.

[29] Hou W, Meng X, Zhao W, et al. Elevated First-Trimester Total Bile Acid is Associated with the Risk of Subsequent Gestational Diabetes. Scientific reports 2016; 6: 34070.

[30] Kong M, Lu Z, Zhong C, et al. A higher level of total bile acid in early mid-pregnancy is associated with an increased risk of gestational diabetes mellitus: a prospective cohort study in Wuhan, China. Journal of endocrinological investigation 2020.

[31] Li J, Huo X, Cao YF, et al. Bile acid metabolites in early pregnancy and risk of gestational diabetes in Chinese women: A nested case-control study. EBioMedicine 2018; 35: 317-24.

[32] Hou W, Meng X, Zhao A, et al. Development of Multimarker Diagnostic Models from Metabolomics Analysis for Gestational Diabetes Mellitus (GDM). Molecular \& cellular proteomics : MCP 2018; 17(3): 431-41.

[33] van Nierop FS, Meessen ECE, Nelissen KGM, et al. Differential effects of a 40-hour fast and bile acid supplementation on human GLP-1 and FGF19 responses. American journal of physiology Endocrinology and metabolism 2019; 317(3): E494-E502.

[34] Qi X, Yun C, Sun L, et al. Gut microbiota-bile acid-interleukin-22 axis orchestrates polycystic ovary syndrome. Nature medicine 2019; 25(8): 1225-33.

\section{Figures}



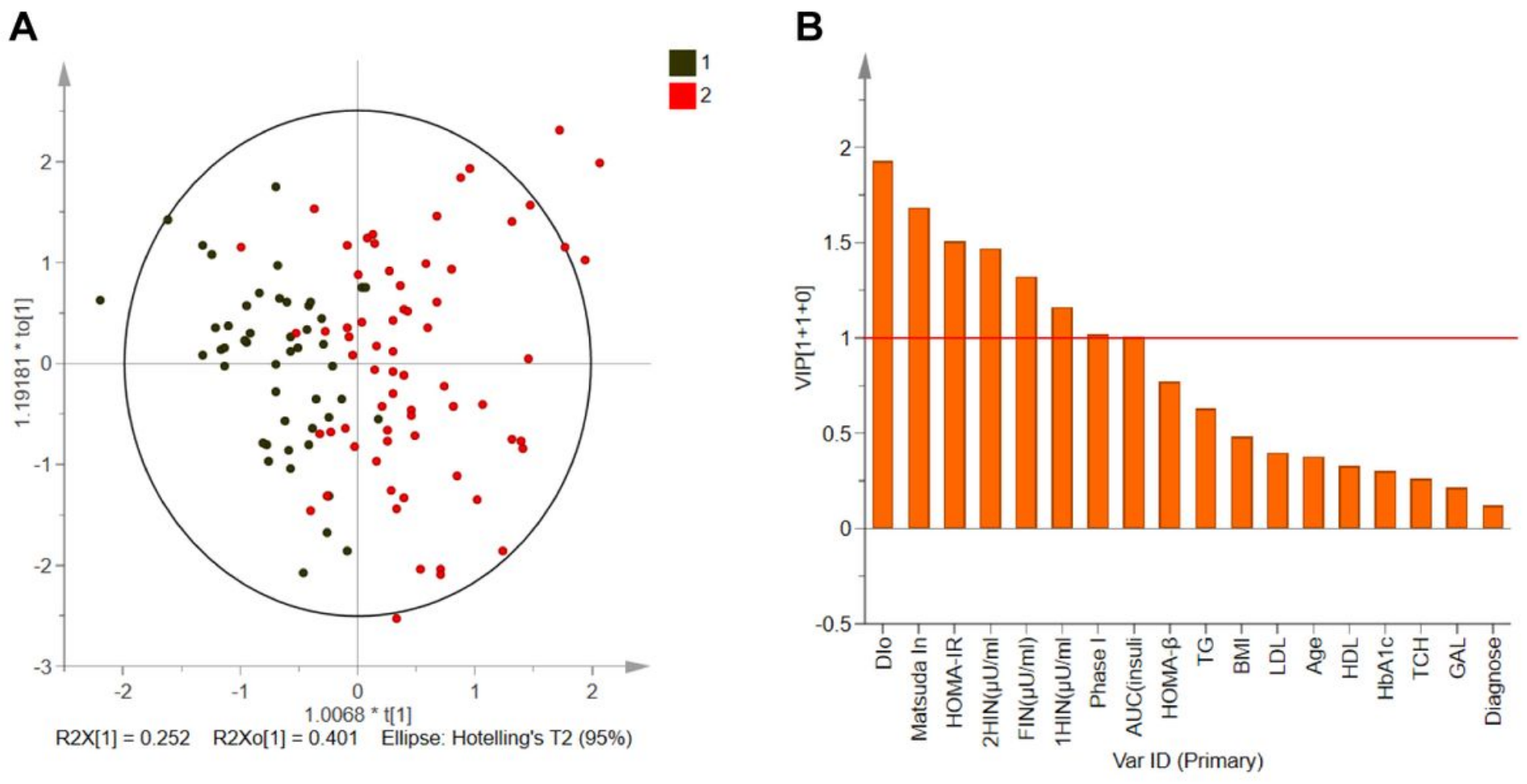

Figure 1

OPLS-DA score plot of NGT and GDM according to the index changed obviously in table 1, the black and red square represent the NGT and GDM individuals respectively, the black circle represents the $95 \%$ confidence interval (A). A taxon with a VIP score of $>1$ was considered important in the group discrimination (B).
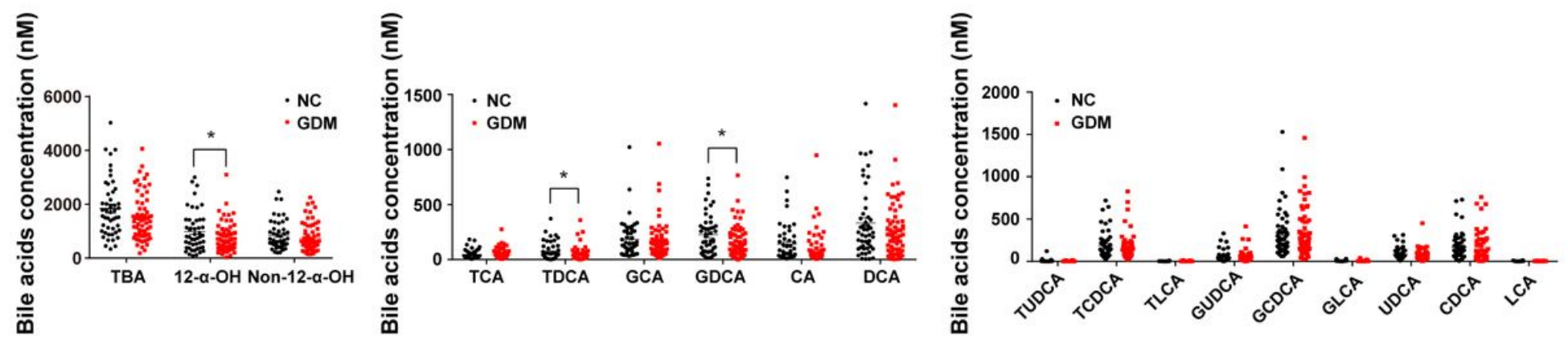

\section{Figure 2}

TBA remained stable in GDM group, 12a-hydroxylated bile acids decreased in GDM (B), non-12ahydroxylated bile acids changed not obviously in GDM group (A). In 12a-hydroxylated bile acids GDCA and TDCA declined significantly in GDM (B), in non-12a-hydroxylated bile acids all the detected individuals exist no obvious variation (C), * $P<0.05$. 


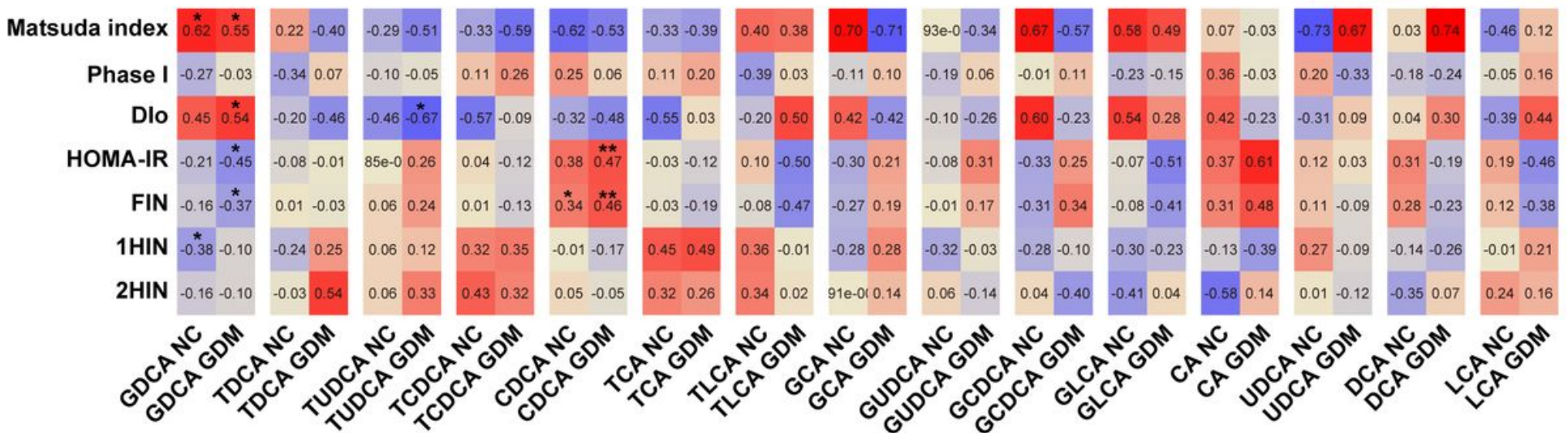

Figure 3

Heat map showing Spearman correlation between bile acids individuals and Matsuda index, Phase I, Dlo, HOMA-IR, FIN, 1HIN, 2HIN. The correlation strength is shown by the color bar, red represents a positive association, blue represents a negative association and white no association, $* P<0.05$, $* * P<0.01$.

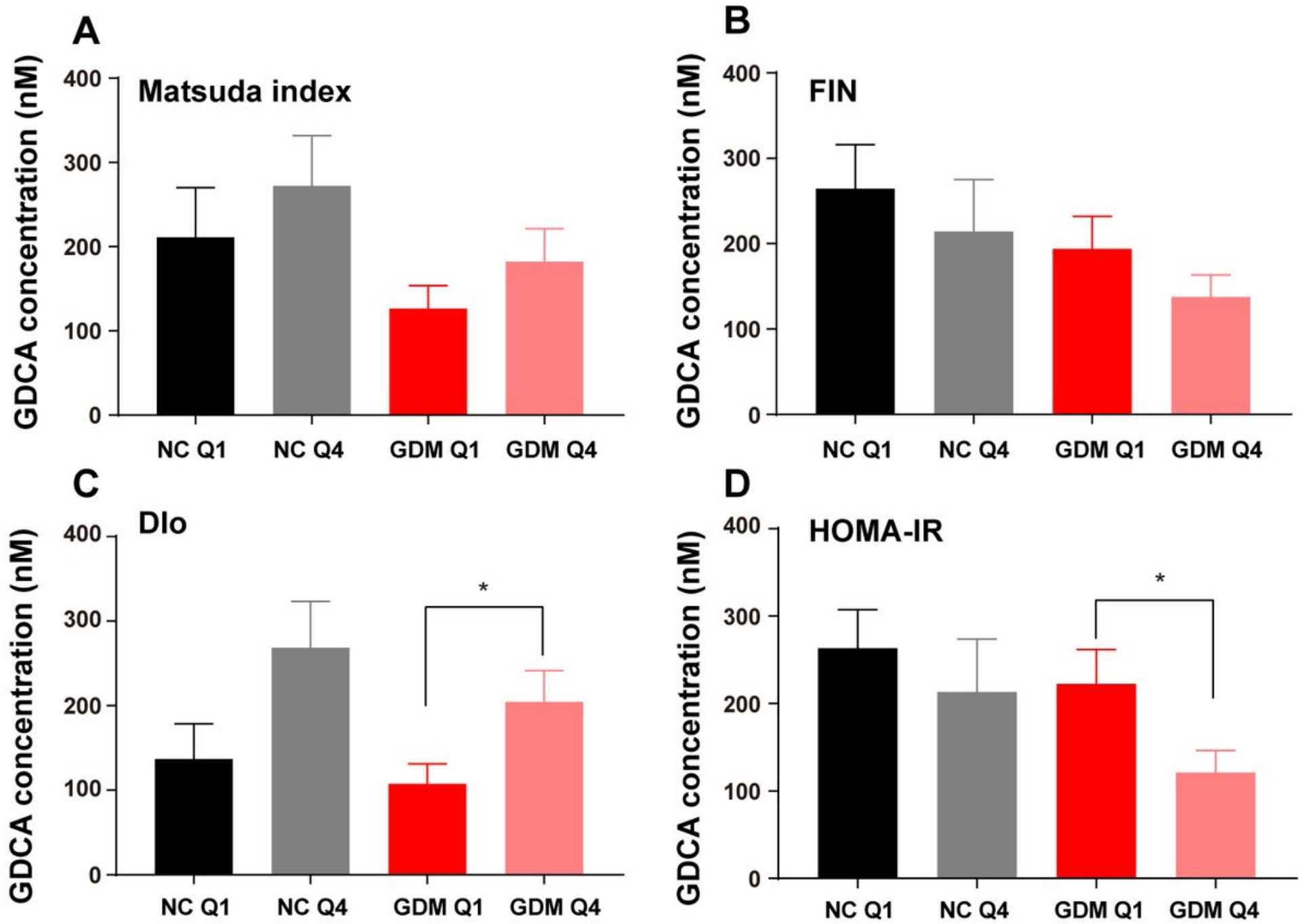

Figure 4 
Change of GDCA concentration in Q1 and Q4 subgroups of NGT and GDM divided by Matsuda index (A), FIN (B), HOMA-IR (C), Dlo (D), * $P<0.05$.

\section{Supplementary Files}

This is a list of supplementary files associated with this preprint. Click to download.

- Tables1.docx

- TableS1.docx 